\title{
THE EILENBERG-MOORE, ROTHENBERG-STEENROD SPECTRAL SEQUENCE FOR $K$ THEORY
}

TED PETRIE

Let $G$ be a topological group and let $G \rightarrow E \rightarrow B_{G}$ be the universal fibration for $G$ so that $B_{G}$ is the classifying space for $G$. Eilenberg and Moore have developed two spectral sequences which deal with this situation [1], [2], [3]. The spectral sequence of Type I has $E_{2}$ $=\operatorname{Cotor}^{* *(G)}(K, K) \Rightarrow H^{*}\left[B_{G}\right]$. The Type II spectral sequence has $E_{2}=\operatorname{Tor}^{H^{*}[B G]}(K, K) \Rightarrow H^{*}[G] . K$ is the ground ring. These tools give a very simple proof of

Theorem $H^{*} . H^{*}[G]$ is an exterior algebra over $K$ iff $H^{*}\left[B_{G}\right]$ is a polynomial ring over $K$.

The Eilenberg-Moore construction of these spectral sequences was almost entirely algebraic [1].

Rothenberg and Steenrod have studied the Type I spectral sequence and have produced a geometric construction which gives rise to it, [4]. Their approach has the advantage that being of geometric origin it applies to other cohomology theories such as the AtiyahHirzebruch complex $K$ theory [5]. In particular, if $G$ is a Lie group and $K^{*}[G]$ is an exterior algebra over $Z$, then one has a spectral sequence $E_{2}=\operatorname{Cotor}^{K^{*}[G]}[Z, Z] \Rightarrow K^{*}\left[B_{G}\right]$. The spectral sequence collapses and hence gives an easy proof of

TheOREM $K^{*}$. If $G$ is a Lie group for which $K^{*}[G]$ is an exterior algebra over $Z$, then $K^{*}\left[B_{G}\right]$, the completed representation ring of $G$, is a power series ring.

The natural question arises as to whether the Type II spectral sequence exists in $K$ theory. The purpose of this note is to show that it does not by showing that the converse of Theorem $K^{*}$ is false.

Counterexample. There does not exist a Moore-Eilenberg spectral sequence of Type II in $K$ theory for the fibration $S O(3) \rightarrow E \rightarrow B_{S O}(3)$.

Proof. $K^{*}[S O(3)]=Z \oplus Z \oplus Z_{2} . K^{*}\left[B_{S O(3)}\right]$ is the completed representation ring of $S O(3)$ which is a power series ring $Z[[\rho]]$. Suppose that such a spectral sequence $E_{2}=\operatorname{Tor}^{K^{*}\left[B_{S O}(3)\right]}[Z, Z] \Rightarrow K^{*}[S O(3)]$

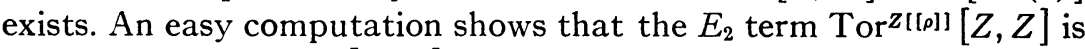
an exterior algebra $E\left[S^{-1} \rho\right]=Z \oplus Z S^{-1} \rho$ generated by the desuspension of $\rho$. Since the $E_{2}$ term $E\left[S^{-1} \rho\right]$ is already "smaller than" $K^{*}[S O(3)]$ we have a contradiction.

Received by the editors October 5, 1966. 
I wish to thank the referee of my paper [6] for pointing out this example in dealing with a related question.

\section{REFERENCES}

1. J. C. Moore, "Algebre homologique et homologie des espaces classifiants," Exposé 7, Séminaire Henri Cartan, 12 ième année: 1959/1960, Periodicité des groupes d'homotopie stables des groupes classiques, d'après Bott, 2nd ed., Secrétariat mathematique, Paris, 1961.

2. S. Eilenberg and J. C. Moore, Homological algebra and fibrations (to appear).

3. - Homology and fibrations. I, Comment. Math. Helv. 40 (1966), 199-236.

4. M. Rothenberg and N. E. Steenrod, The cohomology of classifying spaces of $H$ spaces, Bull. Amer. Math. Soc. 71 (1965), 872-875.

5. M. Atiyah and F. Hirzebruch, Vector-bundles and homogenous spaces, pp. 7-38, Proc. Sympos. Pure Math., Vol. 3, Amer. Math. Soc., Providence, R. I., 1961.

6. T. Petrie, The $K$ theory of the projective unitary groups, Topology 6 (1967), 103-115.

INSTITUTE FOR DEFENSE ANALySES 